\title{
Expansions of Hypergeometric Functions in Hypergeometric Functions
}

\author{
By Jerry L. Fields and Jet Wimp
}

Abstract. In [1] Luke gave an expansion of the confluent hypergeometric function in terms of the modified Bessel functions $I_{\nu}(z)$. The existence of other, similar expansions implied that more general expansions might exist. Such was the case. Here multiplication type expansions of low-order hypergeometric functions in terms of other hypergeometric functions are generalized by Laplace transform techniques.

1. General Expansions. The generalized hypergeometric function ${ }_{p} F_{q}(z),[2]$, is defined by

$$
\left\{\begin{array}{l}
{ }_{p} F_{q}(z)={ }_{p} F_{q}\left(\begin{array}{l}
a_{1}, \cdots, a_{p} \\
b_{1}, \cdots, b_{q}
\end{array} \mid z\right)=\sum_{k=0}^{\infty} \frac{\prod_{j=1}^{p}\left(a_{j}\right)_{k}}{\prod_{j=1}^{q}\left(b_{j}\right)_{k}} \cdot \frac{z^{k}}{k !}, \\
\text { where } \quad(\sigma)_{\mu}=\frac{\Gamma(\sigma+\mu)}{\Gamma(\sigma)} .
\end{array}\right.
$$

We assume that no $a_{j}$ is equal to any $b_{j}$ and that no $b_{j}$ is a negative integer. For ease in writing, we employ the contracted notation

$$
{ }_{p} F_{q}(z)={ }_{p} F_{q}\left(\begin{array}{c}
a_{p} \\
b_{q}
\end{array} \mid z\right)=\sum_{k=0}^{\infty} \frac{\left(a_{p}\right)_{k}}{\left(b_{q}\right)_{k}} \cdot \frac{z^{k}}{k !} .
$$

Thus $\left(a_{p}\right)_{k}$ is to be interpreted as $\prod_{j=1}^{p}\left(a_{j}\right)_{k}$ and similarly for $\left(b_{q}\right)_{k}$. Considered as a power series in $z,{ }_{p} F_{q}(z)$ has a radius of convergence equal to infinity if $p \leqq q$ and equal to unity if $p=q+1$. In general, ${ }_{p} F_{q}(z)$ is not defined if $p \geqq q+2$. However, in this paper, we shall say that ${ }_{p} F_{q}(z w)$ is equal to another series if the coefficients of $(w)^{k}$ on both sides are equal, regardless of the relationship between $p$ and $q$.

Our first expansion is

$$
\begin{aligned}
{ }_{p+r} F_{q+?} & \left(\begin{array}{c}
a_{p}, c_{r} \\
b_{q}, d_{s}
\end{array} \mid z w\right)=\sum_{n=0}^{\infty} \frac{\left(a_{p}\right)_{n}(\alpha)_{n}(\beta)_{n}(-z)^{n}}{\left(b_{q}\right)_{n}(\gamma+n)_{n} n !} \\
& \times{ }_{p+2} F_{q+1}\left(\begin{array}{c}
n+\alpha, n+\beta, n+a_{p} \\
2 n+\gamma+1, n+b_{q}
\end{array} \mid z\right){ }_{r+2} F_{s+2}\left(\begin{array}{c}
-n, n+\gamma, c_{r} \\
\alpha, \beta, d_{s}
\end{array} \mid w\right) .
\end{aligned}
$$

From (1.3) we may derive other expansions by confluence:

$$
\begin{aligned}
{ }_{p+r} F_{q+s}\left(\begin{array}{c}
a_{p}, c_{r} \\
b_{q}, d_{s}
\end{array} \mid z w\right) & =\sum_{n=0}^{\infty} \frac{\left(a_{p}\right)_{n}(\alpha)_{n}(-z)^{n}}{\left(b_{q}\right)_{n}(\gamma+n)_{n} n !} \\
& \times{ }_{p+1} F_{q+1}
\end{aligned}
$$

Received September 30, 1960; revised February 20, 1961. This work was sponsored by the United States Navy through the Applied Mathematics Laboratory of the David W. Taylor Model Basin. 


$$
\begin{array}{r}
{ }_{p+r} F_{q+s}\left(\begin{array}{c}
a_{p}, c_{r} \\
b_{q}, d_{s}
\end{array} \mid z w\right)=\sum_{n=0}^{\infty} \frac{\left(a_{p}\right)_{n}(\alpha)_{n}(-z)^{n}}{\left(b_{q}\right)_{n} n !}{ }_{p+1} F_{q}\left(\begin{array}{c}
\left.n+\alpha, n+a_{p} \mid z\right) \\
n+b_{q}
\end{array} \mid z\right) \\
\times{ }_{r+1} F_{s+1}\left(\begin{array}{c}
-n, c_{r} \mid \\
\alpha, d_{s}
\end{array} \mid w\right) \\
{ }_{p+r} F_{q+s}\left(\begin{array}{c}
a_{p}, c_{r} \\
b_{q}, d_{s}
\end{array} \mid z w\right)=\sum_{n=0}^{\infty} \frac{\left(a_{p}\right)_{n}(-z)^{n}}{\left(b_{q}\right)_{n} n !}{ }_{p} F_{q}\left(\begin{array}{c}
n+a_{p} \mid \\
n+b_{q}
\end{array} \mid z\right) \\
\times{ }_{r+1} F_{s}\left(\begin{array}{c}
-n, c_{r} \\
d_{s}
\end{array} \mid w\right) .
\end{array}
$$

For example, if in (1.3) we replace $r$ by $r+1, z$ by $z / c_{r+1}$, set $\beta=c_{r+1}$ and let $\beta \rightarrow \infty,(1.4)$ results; (1.5) and (1.6) may be similarly derived; (1.6) is a result given previously by Meijer, [7], 1953, page 355, but it is the only result above which can be deduced from his work.

Equation (1.3) is proved by induction on $p, q, r$, and $s$. The case $p=q=r=$ $s=0$ reduces to

$$
e^{z w}=\sum_{n=0}^{\infty} \frac{(\alpha)_{n}(\beta)_{n}(-z)^{n}}{(\gamma+n)_{n} n !}{ }_{2} F_{1}\left(\begin{array}{c}
n+\alpha, n+\beta \\
2 n+\gamma+1
\end{array} \mid z\right){ }_{2} F_{2}\left(\begin{array}{c}
-n, n+\gamma \cdot w \\
\alpha, \beta
\end{array}\right)
$$

a result given by Luke, see (1.8) of [3]. The proof of (1.3) then proceeds by Laplace and inverse-Laplace transform techniques. Assume (1.3) true for $p, q, r$ and $s$, multiply both sides of $(1.3)$ by $(w)^{\sigma-1}$, take the Laplace transform of both sides with respect to $w$, and we obtain, see (17), page 219 of [4],

$$
\begin{array}{r}
\int_{0}^{\infty} e^{-\lambda w} w^{\sigma-1}{ }_{p+r} F_{q+s}\left(\begin{array}{c}
a_{p}, c_{r} \\
b_{q}, d_{s}
\end{array} \mid z w\right) d w=\frac{\Gamma(\sigma)}{(\lambda)^{\sigma}}{ }_{p+r+1} F_{q+s}\left(\begin{array}{c}
a_{p}, c_{r}, \sigma \mid z \\
b_{q}, d_{s}
\end{array}\right) \\
=\frac{\Gamma(\sigma)}{(\lambda)^{\sigma}} \sum_{n=0}^{\infty} \frac{\left(a_{p}\right)_{n}(\alpha)_{n}(\beta)_{n}(-z)^{n}}{\left(b_{q}\right)_{n}(\gamma+n)_{n} n !}{ }_{p+2} F_{q+1}\left(\begin{array}{c}
n+\alpha, n+\beta, n+a_{p} \\
2 n+\gamma+1, n+b_{q}
\end{array} \mid z\right) \\
\times_{r+3} F_{s+2}\left(\begin{array}{c}
-n, n+\gamma, c_{r}, \sigma \mid \frac{1}{\lambda} \\
\alpha, \beta, d_{s}
\end{array}\right) .
\end{array}
$$

The induction on $r$ is completed by replacing $1 / \lambda$ by $w$ in (1.10). The induction with respect to $s$ is effected by multiplying both sides of (1.3) by $(w)^{\sigma}$, letting $w=1 / \lambda$ and applying the inverse-Laplace transform, see (1), page 297 of [4]. If the above Laplace transform techniques are applied to $z$ instead of $w$, the inductions on $p$ and $q$ can be similarly effected.

2. Specialized Expansions. In this section we give several interesting cases of (1.3)-(1.6).

From (1.3) we have

$$
\begin{aligned}
{ }_{r} F_{s}\left(\begin{array}{c}
c_{r} \\
d_{s}
\end{array} \mid z w\right)=\frac{2^{\gamma}}{\left[1+(1-z)^{1 / 2}\right]^{\gamma}} \sum_{n=0}^{\infty} \frac{(\gamma)_{n}(-z)^{n}}{n !\left[1+(1-z)^{1 / 2}\right]^{2 n}} \\
\\
\quad \times{ }_{r+2} F_{s+2}\left(\begin{array}{c}
-n, n+\gamma, c_{r} \\
\gamma / 2,(\gamma+1) / 2, d_{s}
\end{array} \mid w\right),
\end{aligned}
$$

where we have used the relation, see [2], page 101, (6), 


$$
\left[\frac{1}{2}+(1-z)^{1 / 2} / 2\right]^{1-2 a}={ }_{2} F_{1}\left(\begin{array}{c}
a-\frac{1}{2}, a \\
2 a
\end{array} \mid z\right)
$$

The expansion

$$
\begin{aligned}
{ }_{r} F_{s}\left(\begin{array}{c}
c_{r} \\
d_{s}
\end{array} \mid z w\right)=\frac{e^{z / 2}}{\left(\frac{1}{2}\right)_{\gamma}(z)^{\gamma}}\left\{2^{2 \gamma}(1)_{\gamma}\left(\frac{1}{2}\right)_{\gamma} I_{\gamma}(z / 2)\right. \\
\left.\quad+2 \sum_{n=1}^{\infty}(-1)^{n} \frac{(n+\gamma)}{n}(n)_{2 \gamma}{ }_{r+2} F_{s+1}\left(\begin{array}{c}
-n, n+2 \gamma, c_{r} \\
\frac{1}{2}+\gamma, d_{s}
\end{array} \mid w\right) I_{n+\gamma}\left(\frac{z}{2}\right)\right\}
\end{aligned}
$$

follows from (1.4), where $I_{\nu}(z)$ is the modified Bessel function of the first kind. Here, use is made of the expression

$$
\Gamma(\nu+1) I_{\nu}\left(x=(x / 2)^{\nu} e^{-x} F_{1}\left(\begin{array}{c}
\frac{1}{2}+\nu \\
1+2 \nu
\end{array} \mid 2 x\right) .\right.
$$

Also from (1.4) we get

$$
\begin{array}{r}
{ }_{p} F_{q}\left(\begin{array}{l}
a_{p} \\
b_{q}
\end{array} \mid z w\right)={ }_{p+1} F_{q+1}\left(\begin{array}{c}
\beta+1, a_{p} \\
\alpha+\beta+2, b_{q}
\end{array} \mid z\right) \\
+\sum_{n=1}^{\infty} \frac{\left(a_{p}\right)_{n}(z)^{n}}{\left(b_{q}\right)_{n}(n+\alpha+\beta+1)_{n}}{ }^{p+1} F_{q+1}\left(\begin{array}{c}
n+\beta+1, n+a_{p} \\
2 n+\alpha+\beta+2, n+b_{q}
\end{array} \mid z\right) \\
\quad \times P_{n}{ }^{(\alpha, \beta)}(2 w-1),
\end{array}
$$

where

$$
P_{n}{ }^{(\alpha, \beta)}(x)=(-1)^{n} \frac{(1+\beta)_{n}}{n !}{ }_{2} F_{1}\left(\begin{array}{c}
-n, n+\alpha+\beta+1 \mid \frac{1+x}{2} \\
1+\beta
\end{array}\right) .
$$

The $P_{n}{ }^{(\alpha, \beta)}(x)$ are known as the Jacobi polynomials, see [8], and reduce to the Chebyshev polynomials of the first kind if $\alpha=\beta=-\frac{1}{2}$.

Equation (1.5) yields the following expansions:

$$
\begin{gathered}
{ }_{r} F_{s+1}\left(\begin{array}{c}
c_{r} \\
d_{s}, \delta
\end{array} \mid z w\right)=e^{z} \sum_{n=0}^{\infty} \frac{(-z)^{n}}{n !}{ }_{r+1} F_{s+1}\left(\begin{array}{c}
-n, c_{r} \\
\delta, d_{s}
\end{array} \mid w\right) ; \\
{ }_{r} F_{s}\left(\begin{array}{c}
c_{r} \\
d_{s}
\end{array} \mid z w\right)=(1-z)^{-\alpha} \sum_{n=0}^{\infty} \frac{(\alpha)_{n}}{n !}\left(\frac{z}{z-1}\right)^{n}{ }_{r+1} F_{s+1}\left(\begin{array}{c}
-n, c_{r} \\
\alpha, d_{s}
\end{array} \mid w\right) ; \text { and } \\
{ }_{r} F_{s+1}\left(\begin{array}{c}
c_{r} \\
d_{s}, 1+\delta
\end{array} \mid-z w\right)=\frac{\delta}{(z)^{\delta}} \sum_{n=0}^{\infty} \frac{\gamma(\delta+n, z)}{n !}{ }_{r+1} F_{s+1}\left(\begin{array}{c}
-n, c_{r} \\
\delta, d_{s}
\end{array} \mid w\right)
\end{gathered}
$$

where

$$
\left\{\begin{aligned}
\gamma(a, z) & =\Gamma(a)-\int_{z}^{\infty} e^{-t} t^{a-1} d t, \\
& =a^{-1} z^{a}{ }_{1} F_{1}\left(\frac{a}{a+1} \mid-z\right) .
\end{aligned}\right.
$$

$$
\left.{ }_{p} F_{q}\left(\begin{array}{l}
a_{p} \\
b_{q}
\end{array} \mid z w\right)=\sum_{n=0}^{\infty} \frac{\left(a_{p}\right)_{n}(-z)^{n}}{\left(b_{q}\right)_{n}}{ }_{p+1} F_{q}\left(\begin{array}{c}
n+\alpha+1, n+a_{p} \\
n+b_{q}
\end{array}\right) z\right) L_{n}^{\alpha}(w)
$$


where

$$
L_{n}^{\alpha}(x)=\frac{(\alpha+1)_{n}}{n !}{ }_{1} F_{1}\left(\begin{array}{c}
-n \\
1+\alpha
\end{array} \mid x\right) .
$$

The $L_{n}{ }^{\alpha}(x)$ are known as the generalized Laguerre polynomials, see [8].

The result (2.7) can also be deduced from the work of Rainville [5], page 267, (25), and (2.8) is a generalization of a result given by Chaundy [6] and Meijer [7], 1952, page 483.

If we use the formula

$$
{ }_{0} F_{1}\left(\nu+1 \mid \frac{x^{2}}{4}\right)=\Gamma(\nu+1)\left(\frac{2}{x}\right)^{\nu} I_{\nu}(x),
$$

(1.6) yields the expansion

$$
\begin{aligned}
{ }_{r} F_{s+1}\left(\begin{array}{c}
c_{r} \\
d_{s}, 1+\delta
\end{array} \mid \frac{z^{2} w}{4}\right) \\
\quad=\Gamma(1+\delta)\left(\frac{2}{z}\right)^{\delta} \sum_{n=0}^{\infty} \frac{\left(\frac{-z}{2}\right)^{n}}{n !} I_{\delta+n}(z)_{r+1} F_{s}\left(\begin{array}{c}
-n, c_{r} \\
d_{s}
\end{array} \mid w\right) .
\end{aligned}
$$

Since the merits of modified Bessel function expansions are well known, we consider the convergence properties of (2.3) in more detail. They are determined principally by the asymptotic properties of the polynomials

$$
{ }_{r+2} F_{s+1}\left(\begin{array}{c}
-n, n+2 \gamma, c_{r} \\
\frac{1}{2}+\gamma, d_{s}
\end{array} \mid w\right)
$$

for large $n$. For an extensive treatment of the asymptotic properties of these polynomials for large $n$, see [9]. In general, the convergence properties of (2.3) are superior to the original series definition if the polynomials

$$
{ }_{r+2} F_{s+1}\left(\begin{array}{c}
-n, n+2 \gamma, c_{r} \\
\frac{1}{2}+\gamma, d_{s}
\end{array} \mid w\right)
$$

are interpolatory or nearly so. For example, if $r=s, w=1$ and $c_{i}-c_{j}$ is never an integer or zero, for $i \neq j$,

$$
\begin{aligned}
{ }_{r+2} F_{r+1}\left(\begin{array}{c}
-n, n+2 \gamma, c_{r} \\
\frac{1}{2}+\gamma, d_{r}
\end{array} \mid 1\right) & =\left[A n^{2 \mu}+\sum_{t=1}^{r} B_{t} n^{-2 c_{t}}\right] \cdot\left[1+0\left(\frac{1}{n}\right)\right], \\
\mu & =\sum_{t=1}^{r} c_{t}-\sum_{t=1}^{r} d_{t},
\end{aligned}
$$

and the $A$ and $B_{t}$ 's are constants independent of $n$. Incorporating the inequality, see (1), page 49 of [10],

$$
\left|I_{\nu}(z)\right| \leqq \frac{|z / 2|^{\nu}}{\Gamma(\nu+1)} \exp \{\operatorname{Im}(z)\},
$$

we see that (2.3) converges like

$$
\sum_{n=1}^{\infty}\left\{\frac{(n)^{2 \mu+\gamma}}{n ! 4^{n}}+\sum_{t=1}^{r} \frac{n^{\gamma-2 c_{t}}}{n ! 4^{n}}\right\}|z|^{n} .
$$


The original series definition, however, converges like

$$
\sum_{n=1}^{\infty} \frac{n^{\mu}}{n !}|z|^{n}
$$

3. Further Expansions Involving Free Parameters. If in (1.3), we replace $p$ by $p-2$ and set $\alpha=a_{p-1}, \beta=a_{p}, w=0$, we get

$$
1=\sum_{n=0}^{\infty} \frac{\left(a_{p}\right)_{n}(-z)^{n}}{\left(b_{q}\right)_{n}(\gamma+n)_{n} n !}{ }^{p} F_{q+1}\left(\begin{array}{c}
n+a_{p} \\
n+b_{q}, 2 n+\gamma+1
\end{array} \mid z\right) .
$$

Then replacing $a_{p}$ by $a_{p}+k, b_{q}$ by $b_{q}+k, \gamma$ by $\gamma+2 k$ and multiplying both sides of (3.1) by

$$
\frac{\left(c_{r}\right)_{k}(z w)^{k}}{\left(d_{s}\right)_{k} k !}
$$

we get

$$
\begin{array}{r}
\frac{\left(c_{r}\right)_{k}(z w)^{k}}{\left(d_{s}\right)_{k} k !}=\frac{\left(c_{r}\right)_{k}(z w)^{k}}{\left(d_{s}\right)_{k} k !} \sum_{n=0}^{\infty} \frac{\left(k+a_{p}\right)_{n}(-z)^{n}}{\left(k+b_{q}\right)_{n}(n+2 k+\gamma)_{n} n !} \\
\times{ }_{p} F_{q+1}\left(\begin{array}{c}
n+k+a_{p} \\
n+k+b_{q}, 2 n+2 k+\gamma+1
\end{array} \mid z\right) \\
=\sum_{n=k}^{\infty} \frac{\left(a_{p}\right)_{n}(-z)^{n}}{\left(b_{q}\right)_{n}(\gamma+n)_{n} n !}{ }_{p} F_{q+1}\left(\begin{array}{c}
n+a_{p} \\
n+b_{q}, 2 n+\gamma+1
\end{array} \mid z\right) \\
\times \frac{(-n)_{k}(n+\gamma)_{k}\left(b_{q}\right)_{k}\left(c_{r}\right)_{k}(w)^{k}}{\left(a_{p}\right)_{k}\left(d_{s}\right)_{k} k !} .
\end{array}
$$

Summing the terms represented in (3.2) from $k$ equal zero to infinity, and interchanging the summation processes with respect to $k$ and $n$, we obtain

$$
\begin{aligned}
{ }_{r} F_{s}\left(\begin{array}{l}
c_{r} \\
d_{s}
\end{array} \mid z w\right)=\sum_{n=0}^{\infty} \frac{\left(a_{p}\right)_{n}(-z)^{n}}{\left(b_{q}\right)_{n}(\gamma+n)_{n} n !}{ }_{p} F_{q+1} & \left(\begin{array}{c}
n+a_{p} \\
n+b_{q}, 2 n+\gamma+1
\end{array} \mid z\right) \\
& \times{ }_{q+r+2} F_{p+s}\left(\begin{array}{c}
\left.-n, n+\gamma, b_{q}, c_{r} \mid w\right) . \\
a_{p}, d_{s}
\end{array} \mid w\right.
\end{aligned}
$$

Using the Laplace transform techniques of Section 1, we arrive at the expansion

$$
\begin{aligned}
{ }_{r+t} F_{s+u}\left(\begin{array}{c}
c_{r}, e_{t} \\
d_{s}, f_{u}
\end{array} \mid z w\right)=\sum_{n=0}^{\infty} \frac{\left(e_{t}\right)_{n}\left(a_{p}\right)_{n}(-z)^{n}}{\left(f_{u}\right)_{n}\left(b_{q}\right)_{n}(\gamma+n)_{n} n !} \\
\quad \times_{p+t} F_{q+u+1}\left(\begin{array}{c}
n+a_{p}, n+e_{t} \\
n+b_{q}, n+f_{u}, 2 n+\gamma+1
\end{array} \mid z\right) \\
\times_{q+r+2} F_{p+s}\left(\begin{array}{c}
-n, n+\gamma, b_{q}, c_{r} \\
a_{p}, d s
\end{array} \mid w\right) .
\end{aligned}
$$

Equation (3.4) and its confluent form not containing $\gamma$ are generalizations of results given by Chaundy, see (11), page 187 of [2].

As a final example of how Laplace transform techniques may be used in general expansions, we prove 


$$
\begin{aligned}
{ }_{p} F_{q}\left(\begin{array}{l}
a_{p} \\
b_{q}
\end{array} \mid \lambda z\right){ }_{r} F_{s}\left(\begin{array}{c}
c_{r} \\
d_{s}
\end{array} \mid \mu z\right)= & \sum_{n=0}^{\infty} \frac{\left(a_{p}\right)_{n}(\lambda z)^{n}}{\left(b_{q}\right)_{n} n !} \\
& \times{ }_{r+q+1} F_{p+s}\left(\begin{array}{c}
-n, 1-n-b_{q}, c_{r} \\
1-n-a_{p}, d_{s}
\end{array} \mid \frac{(-1)^{p+q+1} \mu}{\lambda}\right) .
\end{aligned}
$$

Again the proof proceeds by induction on $p, q, r$ and $s$. The case $p=q=r=s=0$ reduces to

$$
\begin{aligned}
e^{\lambda z} \cdot e^{\mu z} & =\sum_{n=0}^{\infty} \frac{(\lambda+\mu)^{n} z^{n}}{n !} \\
& =\sum_{n=0}^{\infty} \frac{(\lambda z)^{n}}{n !}{ }_{1} F_{0}\left(-n \mid-\frac{\mu}{\lambda}\right),
\end{aligned}
$$

since

$$
{ }_{1} F_{0}(\alpha \mid z)=(1-z)^{-\alpha} .
$$

Assuming (3.5) true for $p, q, r$ and $s$, the inductions with respect to $r$ and $s$ can be effected by using with respect to $\mu$ the Laplace transform techniques illustrated in the proof of (1.3). The inductions with respect to $p$ and $q$ are similarly carried out with respect to $\lambda$ after making use of the relationship

$$
{ }_{1+p} F_{q}\left(\begin{array}{c}
-n, a_{p} \\
b_{q}
\end{array} \mid z\right)=\frac{\left(a_{p}\right)_{n}(-z)^{n}}{\left(b_{q}\right)_{n}}{ }_{1+q} F_{p}\left(\begin{array}{c}
-n, 1-n-b_{q} \\
1-n-a_{p}
\end{array} \mid \frac{(-1)^{q+p}}{z}\right)
$$

This completes the induction proof. Equation (3.5) is a generalization of results given by Chaundy, see (12)-(15), page $187[2]^{*}$.

Midwest Research Institute

Kansas City, Missouri

1. Y. L. LUKE, "Expansion of the confluent hypergeometric function in series of Bessel functions," $M T A C$, v. 13, 1959, p. 261-271.

2. A. Erdelyi, W. Magnus, F. Oberhettinger \& F. G. Tricomi, Higher Transcendental Functions, McGraw-Hill Book Company, Inc., 1953, Vol. 1. Hereafter in this list, we use the abbreviation H.T.F.

3. Y. L. LUKE \& RichaRd L. Coleman, "Expansion of hypergeometric functions in series of other hypergeometric functions," Math. Comp., v. 15, 1961, p. 233.

4. A. ERDELYI, W. Magnus, F. OberhetTinger \& F. G. Tricomi, Tables of Integral Transforms, Vol. 1, McGraw-Hill Book Company, Inc., 1954.

5. E. D. RAINVILLE, "Certain generating functions and their associated polynomials," Amer. Math. Monthly, v. 52, No. 5, May 1945, or H.T.F., Vol. 3, p. 239-250.

6. T. W. ChaUndy, "An extension of hypergeometric functions," Quart. J. Math. Oxford Ser. 14, 1943, p. 55-78.

7. C. S. MeiJer, "Expansion theorems for the G-function," Indag. Math., v. 14-17, 1952-55.

8. H.T.F., Vol. 2.

9. J. L. FIELDS \& Y. L. LUKE, "Asymptotic expansions of a class of hypergeometric polynomials with respect to the order," Midwest Research Institute Technical Report, July 1, 1959.

10. G. N. Watson, A Treatise on the Theory of Bessel Functions, Cambridge University Press, 1945.

* There are two misprints in these formulas. In (12), $p / q$ should be replaced by $q / p$ and in (13), $-a^{\prime}$ by $a^{\prime}$ on the right side of the equation. 